\title{
STEP 1: un nuovo passo nel controllo del peso
}

\author{
Luca Colangeli $^{1,2} \cdot$ Paolo Sbraccia ${ }^{1,2}$
}

Accettato: 17 giugno 2021 / Pubblicato online: 8 settembre 2021

(c) The Author(s) 2021

\section{Commento a:}

STEP 1 Study Group. Once-weekly semaglutide in adults with overweight or obesity.

J.P. Wilding, R.L. Batterham, S. Calanna, M. Davies,

L.F. Van Gaal, I. Lingvay, B.M. McGowan,

J. Rosenstock, M.T. Tran, T.A. Wadden, S. Wharton,

K. Yokote, N. Zeuthen, R.F. Kushner.

N Eng J Med (2021) 384(11):989

L'aumento di prevalenza di obesità nel mondo rende sempre più urgente la necessità di terapie sicure ed efficaci indirizzate alla perdita di peso e alla riduzione del rischio cardiovascolare.

Recenti metanalisi sui farmaci approvati per il trattamento dell'obesità hanno mostrato un'efficacia in termini di riduzione percentuale del peso corporeo dopo 12 mesi di trattamento corretto per il placebo del $2,9 \%$ per orlistat, del $4,0 \%$ per naltrexone/bupropione e del 5,4\% per liraglutide, risultati piuttosto modesti se paragonati a quelli ottenuti dalla chirurgia bariatrica (per la sleeve gastrectomy, l'intervento più diffuso, i risultati variano tra il 20 e il $30 \%$ ) che, peraltro, garantisce effetti più duraturi nel tempo e una più alta percentuale di remissione di diabete mellito tipo 2 [1].

I risultati dello studio STEP 1, pubblicati di recente sul New England Journal of Medicine, rappresentano un nuovo incoraggiante "passo" verso il futuro del controllo del peso corporeo. Lo studio, un trial clinico multicentrico, randomizzato, in doppio-cieco, ha coinvolto 1961 partecipanti

$\llbracket$ P. Sbraccia

sbraccia@med.uniroma2.it

1 Dipartimento di Medicina dei Sistemi, Università degli Studi di Roma Tor Vergata, Roma, Italia

2 UOC Medicina Interna, Centro Medico dell'Obesità, Policlinico Tor Vergata, Roma, Italia che sono stati assegnati, in un rapporto $2: 1$, a ricevere una somministrazione sottocutanea settimanale di $2.4 \mathrm{mg}$ dell'agonista del recettore di GLP1 (GLP1-RA) semaglutide o placebo per 68 settimane. Entrambi i gruppi hanno ricevuto un intervento sullo stile di vita.

La perdita di peso percentuale media dal baseline è stata di $-14,9 \%$ per il gruppo semaglutide e del $-2,4 \%$ per il gruppo placebo; dunque, una differenza di $-12,4$ punti percentuali a favore di semaglutide.

Inoltre, circa un terzo dei pazienti trattati con semaglutide ha raggiunto addirittura una perdita di peso del $20 \%$, avvicinandosi dunque ai risultati ottenuti dalla chirurgia bariatrica. Contestualmente, i pazienti che hanno ricevuto semaglutide hanno mostrato un miglioramento dei fattori di rischio cardiometabolico (in particolare, un miglior controllo della pressione arteriosa) e anche un incremento nella funzionalità fisica ai questionari di auto-valutazione.

I più comuni eventi avversi sono stati nausea e diarrea, tipicamente transitori e di entità lieve-moderata; tuttavia, una quota maggiore di partecipanti assegnati al gruppo semaglutide rispetto al gruppo placebo ha interrotto precocemente il trattamento $(4,5$ vs $0,5 \%)$.

Volendo imitare i complessi effetti fisiologici indotti dalla chirurgia bariatrica, in particolare sull'asse entero-insulare, una parte consistente della ricerca farmacologica sta volgendo con interesse lo sguardo verso le terapie d'associazione [2]. In questo senso sono promettenti i risultati del trial di fase $1 \mathrm{~b}$ di co-somministrazione di cagrilintide (un analogo a lunga emivita dell' amilina, un ormone pancreatico cosecreto con l'insulina con effetto glucoregolatorio) a diversi dosaggi con semaglutide $2,4 \mathrm{mg}$, senza intervento sullo stile di vita. Lo studio, che si proponeva di valutare come outcome primari gli effetti avversi e la tollerabilità dell'associazione farmacologica, ha mostrato una considerevole perdita di peso di $-15,4 \%$ rispetto al baseline dopo sole 20 setti- 
mane nei pazienti sottoposti a trattamento con cagrilintide $4,5 \mathrm{mg}$ associato a semaglutide $2,4 \mathrm{mg}$, rispetto al $-8,0 \%$ ottenuto dai pazienti trattati con la sola semaglutide, a fronte di una comparabile frequenza di effetti avversi (prevalentemente nausea e vomito, di entità lieve-moderata, con una frequenza comparabile con quella dei precedenti studi sui GLP1-RA) [3].

Questi dati supportano lo sviluppo di nuovi farmaci di combinazione nella terapia dell'obesità e lo studio STEP1 ha confermato che la semaglutide sarà la gamba portante in questo passo verso il futuro.

Funding Note Open access funding provided by Università degli Studi di Roma Tor Vergata within the CRUI-CARE Agreement.

Nota della casa editrice Springer Nature rimane neutrale in riguardo alle rivendicazioni giurisdizionali nelle mappe pubblicate e nelle affiliazioni istituzionali.

Open Access This article is licensed under a Creative Commons Attribution 4.0 International License, which permits use, sharing, adaptation, distribution and reproduction in any medium or format, as long as you give appropriate credit to the original author(s) and the source, provide a link to the Creative Commons licence, and indicate if changes were made. The images or other third party material in this article are included in the article's Creative Commons licence, unless indicated otherwise in a credit line to the material. If material is not included in the article's Creative Commons licence and your intended use is not permitted by statutory regulation or exceeds the permitted use, you will need to obtain permission directly from the copyright holder. To view a copy of this licence, visit http://creativecommons.org/licenses/by/4.0/.

\section{Bibliografia}

1. Tak YJ, Lee SY (2020) Long-term efficacy and safety of anti-obesity treatment: where do we stand? Curr Obes Rep 10(1):14-30

2. Gribble FM, O'Rahilly S (2021) Obesity therapeutics: the end of the beginning. Cell Metab 33(4):705-706

3. Enebo LB, Berthelsen KK, Kankam M et al (2021) Safety, tolerability, pharmacokinetics, and pharmacodynamics of concomitant administration of multiple doses of cagrilintide with semaglutide $2.4 \mathrm{mg}$ for weight management: a randomised, controlled, phase $1 \mathrm{~b}$ trial. Lancet 397(10286):1736-1748 